The Global Health Network Collections • Breastfeeding and Breast Milk - from Biochemistry to Impact, (Ed, Family Larson- Rosenquist Foundation) Georg Thieme Verlag KG

\title{
25 List of Figures
}

Published on: May 01, 2020

Updated on: Mar 31, 2020

DOI: $10.21428 / 3 d 48 c 34 a . c 5 a a b d 26$ 
-Fig. 3.1 Prevalence of breastfeeding at ages up to six months by year (UK, 1995-2010)

Fig. 3.2 Proportion of children who were exclusively breastfed at 3, 4, and 6 months, around year 2005

Fig. 3.3 Trends in exclusive breastfeeding among infants younger than six months

-Fig. 3.4 Rates of exclusive and mixed breastfeeding at discharge in very preterm infants in each study region, and overall national breastfeeding rates (black dots)

Fig. 4.1 Changes in the proportion of infants who were breastfed in developed countries from 1938 to 1980

Fig. 4.2 Social rank of mothers and the proportion of mother's breastfeeding in Australia from 6 weeks to 12 months postpartum in 1983

Fig. 4.3 Thermal images of the breasts of (a) nonlactating and (b) lactating women (red $38^{\circ} \mathrm{C}$, green $\left.31^{\circ} \mathrm{C}\right)$

-Fig. 4.4 A coffee shop advertisement in Perth, Western Australia featuring a mother breastfeeding her 6 month old baby and inviting other breastfeeding mothers to frequent the coffee shop in 2011. (STM 2011, Sunday Times Magazine, January 2016)

Fig. 4.5 Lactating mothers with (a) breast abscess and (b) mastitis. Both mothers breastfed their babies during the breast trauma and for several months after recovery

Fig. 4.6 Sir Astley Cooper author of the seminal book "On the anatomy of the breast" published in 1840

Fig. 4.7 (a) Section of the mammary gland through the nipple, showing ducts over a bristle, unravelled, and proceeding to the posterior part of the gland. (b) A preparation made to show the ligamenta suspensoria supporting the folds of the breast to the inner side of the skin. (c) A view of the gland, dissected and unravelled, to show the ducts over bristles, lobuli, and glandules

-Fig. 4.8 Size of the breast from 11 months to 20years of age. (a) 11 months), (b) 3yr, (c) 4yr, (d) 6yr, (e) $9 \mathrm{yr},(\mathrm{f}) 11 \mathrm{yr},(\mathrm{g}) 12 \mathrm{yr},(\mathrm{h}) 13 \mathrm{yr},(\mathrm{i}) 14 \mathrm{yr},(\mathrm{j}) 16 \mathrm{yr}$ and (k) 20yr

Fig. 4.9 Innervation of the breast. (a) The dorsal of posterior nerve going to the breast (white), (b) The 4th posterior nerve coming out of the chest below the fourth rib, and proceeding to the breast and the nipple 
-Fig. 4.10 Increase in the volume of a breast from preconception to one-month postpartum. (a) photographs, (b) three dimensional computer generated models of the breast

Fig. 4.11 Milk ducts injected with different coloured waxes. (a) showing the radiated direction and inter-ramification of the milk ducts injected with red wax. (b) milk ducts injected with red, yellow, black, green and brown wax with the lobes spread out over a stone. (c) at the lower part of the preparation the separate ducts are seen passing above and beneath each other, to render the breast a cushion; whilst at the upper part the ducts are single, ( $\mathrm{d}$ and e) alveoli six times magnified, ( $\mathrm{f}$ and $\mathrm{g}$ ) alveoli injected with mercury and four times magnified

Fig. 4.12 (a) Arteries (red) and veins (yellow) of the breast from their anterior and posterior sources (b) veins around the nipple, (c) distribution of arteries upon the breast and around the nipple, (d) veins injected in the areola and nipple

Fig. 4.13 Anatomy of the human breast modelled from ultrasound imaging

Fig. 4.14 Milk ducts injected from the nipple. (a) six milk ducts, (b) reservoirs or dilatations of the ducts below the nipple (c) a single lobe

Fig. 4.15 Ultra sound images of the milk ducts below the nipple. No reservoirs or dilatations of the ducts were detected and secretory tissue was present immediately below the nipple

-Fig. 4.16 Drawing by Leonardo da Vinci influenced by Galen's teachings showing a vessel from the uterus to the breast that in fact does not exist

Fig. 4.17 Lymphatic vessels of the female breast ( $\mathrm{a}$ and $\mathrm{b}$ ) lymphatics draining from the nipple to the clavicle. The constrictions in the vessel are the valves in the lymphatic vessels that ensures that the lymph flows away from the breast to the lymph nodes (c) The dense network of lymphatic vessels in the breast List of Figures 39125 - List of Figures 392

Fig. 4.18 Breast volume $(\mathrm{mL})$, a measure of breast growth, and the concentration of human placental lactogen $(\mathrm{mg} / \mathrm{L})$ at three-weekly intervals from conception to birth

Fig. 4.19 Concentration of prolactin $(\mu \mathrm{g} / \mathrm{L})$ in blood and the excretion of lactose $(\mathrm{mmol} / 24 \mathrm{~h})$ in urine at three weekly intervals from conception to birth. Secretory differentiation commences at approximately 18 weeks of pregnancy

Fig. 4.20 Milk production $(\mathrm{mL} / 24 \mathrm{~h})$ in a woman with placental retention from 20 to 44 days postpartum. Dilatation and curettage was carried out at day 23 to remove placental fragments 
-Fig. 4.21 Concentration of progesterone (\% of maximum values) in blood and lactose (\% of maximum values) in mammary secretion from -6 days pre-partum to 5 days postpartum in women and rats

Fig. 4.22 Concentration of progesterone $(\mu \mathrm{g} / \mathrm{L})$ and prolactin $(\mu \mathrm{g} / \mathrm{L})$ in blood of women from birth to 8 days postpartum

Fig. 4.23 Circadian changes in the concentration of prolactin $(\mu \mathrm{g} / \mathrm{L})$ in the blood plasma of 8 normal women

Fig. 4.24 Concentration of prolactin $(\mu \mathrm{g} / \mathrm{L})$ in the blood plasma of breastfeeding women from 60 minutes before to 180 minutes after the commencement of breastfeeds

Fig. 4.25 Concentration of prolactin ( $\mu \mathrm{g} / \mathrm{L})$ in the blood plasma of 11 lactating women at 1, 2, and 4, and 6 months of lactation. Blood samples were taken immediately before and 45 minutes after the commencement of the breastfeed

Dig. 4.26 Milk production $(\mathrm{mL} / 24 \mathrm{~h})$ and the concentrations of lactose $(\mathrm{mM})$, total protein $(\mathrm{g} / \mathrm{L})$, citrate $(\mathrm{mM})$ and sodium $(\mathrm{mM})$ in mammary secretion from day 1 to day 5 of lactation, that is, during secretory activation

Fig. 4.27 Milk production (mL/24h) in three groups of mothers 33 to 38 pre-term preterm from birth to 14 days postpartum. One group used an experimental suction pattern that was designed to simulate the baby sucking, another group received the experimental pattern until secretory activation ( $80 \mathrm{~h}$ postpartum) and then the standard pattern and the final group only received the standard pattern

Fig. 4.28 Myoepithelial cells surrounding contracted alveoli from the mammary gland of a lactating goat

Fig. 4.29 Ultrasound image of a milk duct (a) prior to milk ejection and, (b) one minute after milk ejection. White flecks in the ducts in the mage $(b)$ are fat globules

Fig. 4.30 Rate of milk flow and accumulated weight of milk in left and right breasts during breast expression. The peaks in milk flow relate to the number of milk ejections that occurred during the expression period

Fig. 4.31 Sagittal mid-line images of an infant's oral cavity during breastfeeding showing stylised overlay of ultrasound images showing the soft palate, hard palate, nipple and tongue (a) tongue up (baseline vacuum) (b) tongue down (peak vacuum)

Fig. 4.32 Simultaneous recordings of infant intra-oral vacuum and respiration (respiratory inductive plethysmography, RIP) during a breastfeed. The intra-oral vacuum shows a variable baseline vacuum 
(latch vacuum) and a peak vacuum (sucking vacuum). The respiratory trace measures respiration as inspiration effort and expiration effort and absence of a signal indicates a swallow. The inspiratory phase of swallowing can be identified (E-S-I, expiration-suck-inspiration; I-S-I, inspiration-suckinspiration)

-Fig. 4.33 Volume of milk consumed at each breastfeed from left and right breasts over a period of $24 \mathrm{~h}$, (30\% of babies consistently fed from only one breast at each breastfeed and only $13 \%$ of babies fed from both breasts at each breastfeed; $\mathrm{n}=70$ )

Fig. 4.34 (a) Changes in breast volume for each breast at each breastfeed over a 24 h period. (b) The rate of milk synthesis between each breastfeed for each breast over a $24 \mathrm{~h}$ period

-Fig. 4.35 Part of a lobule from the left half of the mammary gland of a lactating goat fixed while distended with milk (a). The right half of the mammary gland of the same goat which was milked out as completely as possible before autopsy (b); note the contracted lobules with collapsed alveoli and ducts lined with a thick folded epithelium

Fig. 4.36 Death of babies in summer from diarrhoea 1895-1904. Deaths of babies in Summer from diarrhoea and high incidence of tuberculosis in army recruits. Prompted the Government to establish Child Health Nurses who were trained by 25 - List of Figures List of Figures 393 free immigrants who, in turn, learnt hygiene on sailing boats coming to Australia

Fig. 4.37 Serial samples of breast milk collected during breast expression The samples were centrifuged to separate the cream showing the increase in the fat content of breast milk from a low concentration in milk from a full breast and a higher concentration of fat in milk from a drained breast

Fig. 4.38 Reference ranges for the growth of breastfed boys

Fig. 4.39 Reference ranges for the growth of breastfed girls

Fig. 4.40 Concentration of lactose (\% of day zero value) in the mammary secretion of (a) women, (b) cow, (c) sows, and (d) rats from 0 to 30 days after removal of milk had ceased

Fig. 4.41 Relative change in breast volume $(\mathrm{mL})$ from pre-conception (relative volume, zero), through pregnancy, lactation and weaning

Fig. 5.1 Protein content of mammalian milks relative to time to doubling of offspring weight. Note the low human protein milk content in humans with relatively slow offspring growth

Fig. 5.2 Decrease in milk protein intake in a breastfed human infant in the first six months reflecting the decrease in infant growth rate. Milk protein intake is calculated as $75 \%$ of crude protein intake 
Fig. 5.3 Growth (standard deviation scores of weight for length) of breast and bottle fed infants up to the age of 24 months

Fig. 5.4 Lower risk of overweight and obesity at early school age among more than 9,000 children in Bavaria, Germany (adjusted for confounding variables) in children ever breastfed versus never breastfed

Fig. 7.1 Freshly isolated human milk cells stained with Trypan Blue for cell viability. Cells stained dark blue indicate dead or dying cells

Fig. 7.2 Changes in HM immune cell (CD45 +) content from colostrum to week 10 postpartum, and between HM samples collected from healthy and infected mother-infant dyads. The two cell photographs show freshly isolated cells from HM, and a HM immune cell stained for CD45. Maternal and infant infections stimulate a rapid leukocyte response in breast milk

Fig. 7.3 The current model of miRNA biogenesis and the proposed model of gene expression regulation. RNA polymerase II/III processes primary miRNA (pri-miRNA) from either independent specific genes (miRNA genes) or from introns (protein-coding genes). Inside the nucleus, precursor miRNA (pre-miRNA) is processed from pri-miRNA by the Drosha-DGCR8 complex. Then, pre-miRNA is transported to the cytoplasm by Exportin 5, where Dicer processes the miRNA duplex. Only one strand of the miRNA duplex (called mature miRNA) is attached to the RNA-induced silencing complex (RISC), which can bind its target (mRNA) for either translation repression or mRNA

Fig. 11.1 A schematic representation of the NetBenefit (utility) Maximisation model of breastfeeding decision proposed by Racine et al.

Fig. 11.2 A schematic of evidence hierarchy on benefits of breastfeeding to infants and children in industrialised countries based on Allen \& Hector. Corroborated conclusions by Renfrew et al. are shown in bold

Fig. 12.1 Cup feeding an infant

Fig. 12.2 Feeding with a paladai

Fig. 12.3 Nipple shield

Fig. 12.4 Bolivian aguayo

Fig. 12.5 Brazilian preterm Infant in kangaroo care and receiving breast milk

Fig. 16.1 (a) Sample laminated instruction card for mothers to use in the NICU (neonatal intensive care unit) to disinfect the exterior of the breast pump prior to use. (b) The nurse shows the card, 
attached to the breast pump, to the mother as she demonstrates the disinfection procedure

Fig. 16.2 Sample parent education sheet that reinforces staff teaching about proper care of breast pump collection equipment

Fig. 16.3 The nurse has prepared the infant's 2- hourly bolus feeding and is placing it in the incubator approximately 1 hour prior to feeding so that it can warm (without water) to approximately body temperature

-Fig. 16.4 The mother has used a breast pump to remove most of her human milk (HM) and then the infant is placed at breast to "taste" HM just prior to and during the gavage feeding

-Fig. 16.5 Mean daily intake of HM (human milk) by breast and bottle for 24 premature infants discharged from the NICU (neonatal intensive care unit) at a mean of $36 \pm 2$ weeks PMA (postmenstrual age). This graphic illustrates that while infants have access to an adequate volume of HM each day, they are not able to remove it from the breast 25 - List of Figures 394 effectively until achieving approximately 40-44 weeks PMA

Fig. 16.6 Use of breastfeeding positions that support the infant's head and neck can help to compensate for weak intraoral suction pressures

Fig. 16.7 Use of ultrathin nipple shields can help to compensate for weak intraoral suction pressures, thus facilitating HM intake during breastfeeding in premature infants

Fig. 18.1 Overview of possible treatment methods for human milk to reduce microbial contamination

Fig. 20.1 Transcellular distribution of drugs across the lactocyte

Fig. 22.1 Exclusive breastfeeding (EBF) prevalence among infants < 6 months of age, globally and by world region

-Fig. 22.2 Complex Adaptive Systems key constructs

Fig. 22.3 AIDED scaling-up framework. Specific descriptions of each of the AIDED framework components are provided in the text

Fig. 22.4 Breastfeeding Gear Model

Fig. 22.5 Application of the Breastfeeding Gear Model for understanding differences in breastfeeding performance between Brazil and Mexico 